\title{
Towards a Theory of Differential Constraints of a Hydrodynamic Hierarchy
}

\author{
L MARTÍNEZ ALONSO ${ }^{\dagger}$ and A B SHABAT ${ }^{\ddagger}$ \\ † Departamento de Física Teórica II, Universidad Complutense, E28040 Madrid, Spain \\ E-mail: luism@fis.ucm.es \\ ‡ Landau Institute for Theoretical Physics, RAS, Moscow 117 334, Russia \\ E-mail: shabat@itp.ac.ru
}

Received July 9, 2002; Revised October 2, 2002; Accepted October 3, 2002

\begin{abstract}
We present a theory of compatible differential constraints of a hydrodynamic hierarchy of infinite-dimensional systems. It provides a convenient point of view for studying and formulating integrability properties and it reveals some hidden structures of the theory of integrable systems. Illustrative examples and new integrable models are exhibited.
\end{abstract}

\section{Introduction}

In this paper we are concerned with the hierarchy of evolution equations

$$
\frac{\partial G}{\partial t_{i}}=\left\langle A_{i}, G\right\rangle, \quad G=G(\lambda, x, t), \quad \boldsymbol{t}:=\left(t_{1}, t_{2}, \ldots\right), \quad i \geq 1,
$$

where $\langle U, V\rangle:=U V_{x}-U_{x} V$, and the function $G$ is assumed to posses an expansion

$$
G=g_{0}(x, \boldsymbol{t})+\frac{g_{1}(x, \boldsymbol{t})}{\lambda}+\frac{g_{2}(x, \boldsymbol{t})}{\lambda^{2}}+\cdots, \quad \lambda \rightarrow \infty,
$$

We consider two different forms of (1.1) corresponding to the cases $g_{0} \equiv 1$ and $g_{0} \not \equiv$ const, which will be henceforth referred to as the normalized $G^{(n)}$ and the Schwarzian $G^{(s)}$ hierarchies, respectively. They are determined by two different definitions of the functions $A_{i}=A_{i}(\lambda, x, \boldsymbol{t})$; namely,

$$
A_{i}^{(n)}=\lambda^{i}+\cdots+g_{i-1} \lambda+g_{i}, \quad A_{i}^{(s)}=g_{0} \lambda^{i}+\cdots+g_{i-1} \lambda,
$$

respectively. In both cases we may write

$$
A_{i}=\left(\lambda^{i} G\right)_{+}, \quad i \geq 1,
$$


where the projectors $P: A \rightarrow A_{+}$acting on power series $A=\sum_{n} a_{n} \lambda^{n}$ is defined as

$$
A_{+}^{(n)}:=P^{(n)}(A)=\sum_{n \geq 0} a_{n} \lambda^{n}, \quad A_{+}^{(s)}:=P^{(s)}(A)=\sum_{n \geq 1} a_{n} \lambda^{n} .
$$

In terms of the coefficients of the expansion of $G$, the hierarchy (1.1) becomes a set infinite-dimensional systems of hydrodynamic type

$$
\begin{aligned}
\partial_{i} g_{n} & =\sum_{k=1}^{n}\left\langle g_{n-k}, g_{i+k}\right\rangle, & n \geq 0, & \text { (normalized case) }, \\
\partial_{i} g_{n} & =\sum_{k=0}^{n}\left\langle g_{n-k}, g_{i+k}\right\rangle, & n \geq 0, & \text { (Schwarzian case). }
\end{aligned}
$$

We will show below that the case $g_{0} \not \equiv$ const can be considered as the Schwarzian form of the case $g_{0} \equiv 1$ and that a transformation connecting both cases exists.

Although equations similar to (1.1) were considered in the literature [1, 2, 3, 4] (starting with [1]), the normalized case of the hierarchy (1.1) was recently reconsidered in [5] from the point view of the reduction theory of integrable systems. It was in this context where the notion of differential constraint was introduced. One of the main aims of the present work is to show that this notion is a useful one in the theory of integrable systems.

For several purposes it is convenient to use instead of $G$ the function

$$
\begin{aligned}
& H:=\frac{1}{G}=h_{0}+\frac{h_{1}}{\lambda}+\frac{h_{2}}{\lambda^{2}}+\cdots, \\
& h_{0}=\frac{1}{g_{0}}, \quad h_{1}=-\frac{g_{1}}{g_{0}^{2}}, \quad h_{2}=\frac{g_{1}^{2}}{g_{0}^{3}}-\frac{g_{2}}{g_{0}^{2}}, \quad \ldots
\end{aligned}
$$

It allows us to write (1.1) in the alternative form

$$
\frac{\partial H}{\partial t_{i}}=\frac{\partial}{\partial x}\left(A_{i} H\right)
$$

which implies that the coefficients of the expansion of $H$ in powers of $\lambda$ supply an infinite set of conservation laws for (1.1).

The function $H$ is particularly useful to deal with the reductions of (1.1) determined by differential constraints. Let us illustrate this fact by considering the first flow $t:=t_{1}$ of the Schwarzian hierarchy, which according to (1.4) reads

$$
h_{n, t}=\hat{h}_{n+1, x}, \quad \hat{h}_{n}:=\frac{h_{n}}{h_{0}}, \quad n \geq 0 .
$$

Let us look for a differential constraint of the form

$$
\hat{h}_{1}=B\left(h_{0}, h_{0, x}\right),
$$

allowing to reduce the first equation in (1.5) to

$$
h_{0, t}=D_{x} B\left(h_{0}, h_{0, x}\right) \text {, }
$$


where $D_{x}$ stands for the total differentiation with respect to $x$. The differential constraint (1.6) should be compatible with the whole hierarchy. In particular, the second equation in (1.5) implies

$$
D_{t}\left(h_{1}\right)=D_{t}\left(h_{0} B\left(h_{0}, h_{0, x}\right)\right) \in \operatorname{Im} D_{x} .
$$

Here $D_{t}$ is the total differentiation operator with respect to $t$ and $\operatorname{Im} D_{x}$ denotes the range of $D_{x}$ acting on the set of polynomials in the variables $h_{0}, h_{0, x}, h_{0, x x}, \ldots$ Now one easily finds that

$$
D_{t}\left(h_{1}\right) \equiv-\left(h_{0} B_{11} B_{1}\right) h_{0, x x}^{2}+\cdots,
$$

where $B_{11}$ is the second derivative of $B$ with respect to the argument $h_{0, x}$. Thus (1.8) yields $B_{11}=0$ and therefore the constraint (1.6) should be of the form

$$
\hat{h}_{1}=b\left(h_{0}\right)+a\left(h_{0}\right) h_{0, x} .
$$

One can directly prove in this way that the only constraint (1.6) compatible with (1.5)) is

$$
\left(\log h_{0}\right)_{x}=h_{1} .
$$

The corresponding reduced evolution equation (1.7) is given by

$$
h_{0, t}+\left(\frac{1}{h_{0}}\right)_{x x}=0 .
$$

Several interesting generalizations of this type of constraints will be discussed in this paper. For example it will be proved that the constraints

$$
\left(\log h_{0}\right)_{x}=h_{m}, \quad m \geq 1,
$$

and

$$
\left\{h_{0}, x\right\}=\left[H^{2}\right]_{n}, \quad n \geq 1,
$$

are compatible with (1.5). Here

$$
\{a, x\}:=\frac{3}{4}\left(\frac{a_{x}}{a}\right)^{2}-\frac{1}{2} \frac{a_{x x}}{a} .
$$

and $\left[H^{2}\right]_{n}$ stands for the coefficient at $\lambda^{-n}$ of the expansion of $H^{2}$ in powers of $\lambda$.

The paper is organized as follows. In Section 2 we deal with the structure of symmetries of the hierarchies (1.1) and reformulate some basic results. A particular attention is devoted to applying the classical Liouville method of solution for solving the polynomial reductions of (1.1). Some apparently new results concerning $\tau$-functions like objects (Theorem 2) and a map $G^{(s)} \rightarrow G^{(n)}$ (Theorem 3) are also presented. Section 3 is concerned with the use of the method of differential constraints to characterize nonlinear integrable models from (1.1). It is shown how the structure of wide classes of these models as well as simple strategies for finding solutions can be conveniently described by this method. Some new integrable models, including a $2+1$-dimensional one (3.22) are exhibited. 


\section{Symmetries}

We first reformulate some already known basic properties about the symmetries of the hierarchy (1.1). Our starting point is the following theorem [5]

Theorem 1. The evolutionary flows defined by (1.1) form a commuting family.

Proof. By using the Jacobi identity for the wronskian operation $\langle U, V\rangle$ we have

$$
\frac{\partial^{2} G}{\partial t_{i} \partial t_{j}}-\frac{\partial^{2} G}{\partial t_{j} \partial t_{i}}=\left\langle\frac{\partial A_{j}}{\partial t_{i}}-\frac{\partial A_{i}}{\partial t_{j}}+\left\langle A_{j}, A_{i}\right\rangle, G\right\rangle .
$$

¿From (1.1) and taking into account that $A_{i}=\left(\lambda^{i} G\right)_{+}$it follows

$$
\frac{\partial A_{j}}{\partial t_{i}}-\frac{\partial A_{i}}{\partial t_{j}}=\left(\left\langle A_{i}, \lambda^{j} G\right\rangle-\left\langle A_{j}, \lambda^{i} G\right\rangle\right)_{+} .
$$

On the other hand

$$
\left\langle A_{j}, \lambda^{i} G\right\rangle=-\left\langle\lambda^{i} G, \lambda^{j} G-\left(\lambda^{j} G\right)_{-}\right\rangle=\left\langle\lambda^{i} G,\left(\lambda^{j} G\right)_{-}\right\rangle,
$$

where $\left(\lambda^{j} G\right)_{-}:=\lambda^{j} G-\left(\lambda^{j} G\right)_{+}$. Hence

$$
\begin{aligned}
\frac{\partial A_{j}}{\partial t_{i}}-\frac{\partial A_{i}}{\partial t_{j}} & =\left(\left\langle A_{i}, \lambda^{j} G\right\rangle-\left\langle\lambda^{i} G,\left(\lambda^{j} G\right)_{-}\right\rangle\right)_{+}=\left(\left\langle A_{i}, \lambda^{j} G\right\rangle-\left\langle A_{i},\left(\lambda^{j} G\right)_{-}\right\rangle\right)_{+} \\
& =\left\langle A_{i}, A_{j}\right\rangle_{+}=\left\langle A_{i}, A_{j}\right\rangle
\end{aligned}
$$

which proves the statement.

The hydrodynamic character of the equations of the hierarchy (1.1) has important consequences. Thus, it follows that if $\lambda=\lambda_{0}$ is either a zero or a pole of $G$ then

$$
\frac{\partial \lambda_{0}}{\partial t_{i}}=A_{i}\left(\lambda_{0}, x, \boldsymbol{t}\right) \frac{\partial \lambda_{0}}{\partial x}
$$

It means that zeros and poles of $G$ are Riemann invariants of (1.1). This is a useful property because an obvious reduction of (1.1) arises when only a finite number of coefficients in the expansion (1.2) of $G$ are different from zero

$$
G^{(n)}=1+\frac{g_{1}}{\lambda}+\cdots+\frac{g_{N}}{\lambda^{N}}, \quad G^{(s)}=g_{0}+\frac{g_{1}}{\lambda}+\cdots+\frac{g_{N-1}}{\lambda^{N-1}} .
$$

In this case it is possible to perform the integration of (1.1) in terms of Riemann invariants. Let us illustrate this feature for the normalized case. We can rewrite $G$ in the form

$$
G=\frac{1}{\lambda^{N}} \prod_{i=1}^{N}\left(\lambda+\gamma_{i}(x, \boldsymbol{t})\right)
$$

where $\gamma_{i}(x, \boldsymbol{t})$ are the zeros of $G$. Thus, under the change of dependent variables

$$
\vec{g}:=\left(g_{1}, \ldots, g_{N}\right) \mapsto \vec{\gamma}:=\left(\gamma_{1}, \ldots, \gamma_{N}\right),
$$


the system (1.1) reduces to

$$
\partial_{n} \gamma_{i}=\Omega_{n i}(\vec{\gamma}) \partial_{x} \gamma_{i}, \quad n \geq 1 .
$$

Here $\Omega_{n i}:=\left.A_{n}\right|_{\lambda=-\gamma_{i}}$ are given by

$$
\Omega_{n i}= \begin{cases}(-1)^{n} \sum_{j_{l} \neq i} \gamma_{j_{1}} \gamma_{j_{2}} \cdots \gamma_{j_{n}} & \text { if } n=1,2, \ldots, N-1, \\ 0 & \text { if } n \geq N .\end{cases}
$$

The equations (2.4)-2.5) form a set of $N-1$ weakly nonlinear hydrodynamic systems of Dubrovin type 2 which can be integrated by means of a version of the classical Liouville method [6] proposed by Tsarev [7] (see also [8]). It basically consists in finding a vector field $D_{x}$ acting on the new dependent variables $\vec{\gamma}$

$$
D_{x} \gamma_{i}=X_{i}(\vec{\gamma}), \quad i=1, \ldots, N,
$$

in such a way that it commutes with the fields $\partial_{j}, j=1, \ldots, N-1$. This is verified provided

$$
\frac{\partial \ln X_{i}}{\partial \gamma_{j}}=\frac{1}{\gamma_{i}-\gamma_{j}}, \quad i \neq j .
$$

By applying Tsarev's method (see [7, 8]) one finds

Lemma 1. A general solution of (2.7) is

$$
D_{x} \gamma_{i}=\frac{a_{i}\left(\gamma_{i}\right)}{\prod_{j \neq i}\left(\gamma_{i}-\gamma_{j}\right)}, \quad i=1, \ldots, N,
$$

where $a_{i}(\xi)$ are $N$ arbitrary functions.

As a consequence we can reformulate (2.4) as a set of $N$ dynamical systems for $\gamma_{i}$

$$
\begin{aligned}
& \partial_{n} \gamma_{i}=\Omega_{n i} X_{i}, \quad n=1, \ldots, N-1, \\
& \partial_{x} \gamma_{i}=X_{i} .
\end{aligned}
$$

Equivalently, we have

$$
\frac{\mathrm{d} \gamma_{i}}{a_{i}\left(\gamma_{i}\right)}=\frac{2}{\prod_{j \neq i}\left(\gamma_{i}-\gamma_{j}\right)}\left(\mathrm{d} x+\sum_{n=1}^{N-1} \Omega_{n i} \mathrm{~d} t_{n}\right), \quad i=1, \ldots, N
$$

One can explicitly invert (2.10) (see [8]) and find

$$
(-1)^{n} \mathrm{~d} t_{n}=\sum_{i=1}^{N} \frac{\gamma_{i}^{N-n-1}}{a_{i}\left(\gamma_{i}\right)} \mathrm{d} \gamma_{i}, \quad n=0, \ldots, N-1,
$$

where we are denoting $t_{0}:=x$. Therefore, we conclude that the general solution of (2.4) is determined by the following system of $N$ implicit relations

$$
(-1)^{n} t_{n}=\sum_{i=1}^{N} \int^{\gamma_{i}} \frac{\gamma_{i}^{N-n-1}}{a_{i}\left(\gamma_{i}\right)} \mathrm{d} \gamma_{i}, \quad n=0, \ldots, N-1 .
$$

The theorems which follow state some apparently new results on the structure of the hierarchy (1.1) 
Theorem 2. The differential forms

$$
\begin{array}{ll}
\omega_{1}=g_{1} \mathrm{~d} x+g_{2} \mathrm{~d} t_{1}+\cdots+g_{n} \mathrm{~d} t_{n+1}+\cdots, & \text { (normalized case) }, \\
\omega_{2}=\frac{1}{g_{0}}\left(\mathrm{~d} x-g_{1} \mathrm{~d} t_{1}+\cdots-g_{n} \mathrm{~d} t_{n}+\cdots\right), & (\text { Schwarzian case }),
\end{array}
$$

are closed.

Proof. Let us consider the normalized case. Notice that

$$
\omega_{1}=\sum_{i \geq 0} \operatorname{Res}\left(\lambda^{i} G\right) \mathrm{d} t_{i},
$$

where $\operatorname{Res}(A(\lambda))$ stands for the coefficient of $\lambda^{-1}$ of Laurent series $A(\lambda)$ in $\lambda$. Moreover, from (1.1)

$$
\frac{\partial}{\partial t_{j}} \operatorname{Res}\left(\lambda^{i} G\right)=\operatorname{Res}\left(\lambda^{i} \frac{\partial G}{\partial t_{j}}\right)=\operatorname{Res}\left(\left\langle A_{j}, \lambda_{i} G\right\rangle\right) .
$$

On the other hand

$$
\begin{aligned}
\operatorname{Res}\left(\left\langle A_{j}, \lambda_{i} G\right\rangle\right) & =\operatorname{Res}\left(\left\langle\lambda^{j} G-(\lambda G)_{-}, \lambda_{i} G\right\rangle\right)=-\operatorname{Res}\left(\left\langle\left(\lambda^{j} G\right)_{-},\left(\lambda_{i} G\right)_{+}\right\rangle\right) \\
& =\operatorname{Res}\left(\left\langle A_{i},\left(\lambda_{j} G\right)_{-}\right\rangle\right)=\operatorname{Res}\left(\left\langle A_{i}, \lambda_{j} G\right\rangle\right)
\end{aligned}
$$

so that (2.13) implies

$$
\frac{\partial}{\partial t_{j}} \operatorname{Res}\left(\lambda^{i} G\right)=\frac{\partial}{\partial t_{i}} \operatorname{Res}\left(\lambda^{j} G\right),
$$

which proves that $\omega_{1}$ is closed.

The proof for the Schwarzian case can be done similarly by writing

$$
\omega_{2}=\frac{1}{g_{0}}-\sum_{i \geq 1} \operatorname{Res}\left(\lambda^{i-1} \frac{G}{g_{0}}\right) \mathrm{d} t_{i},
$$

and by taking into account that

$$
\frac{\partial}{\partial t_{i}} \frac{1}{g_{0}}=-\frac{\partial}{\partial x} \frac{g_{i}}{g_{0}}
$$

As a consequence of this theorem there exist two functions $q_{i}=q_{i}(x, \boldsymbol{t})$ verifying

$$
\omega_{i}=\mathrm{d} q_{i}, \quad i=1,2,
$$

so that we can rewrite the hierarchy (1.1) as a system of partial differential equations for $q_{i}$. 
Theorem 3. Let $G^{(s)}=G^{(s)}(\lambda, x, t)$ be a solution of the Schwarzian hierarchy (1.1). If we define $\hat{x}=q_{2}(x, \boldsymbol{t})$, where $q_{2}$ satisfies (2.15), then

$$
G^{(n)}(\lambda, \hat{x}, \boldsymbol{t}):=\frac{G^{(s)}(\lambda, x, \boldsymbol{t})}{g_{0}(x, \boldsymbol{t})}
$$

solves the normalized hierarchy (1.1).

Proof. Observe that

$$
\frac{\partial \hat{x}}{\partial x}=\frac{1}{g_{0}}, \quad \frac{\partial \hat{x}}{\partial t_{i}}=\frac{1}{g_{i}}, \quad i \geq 1 .
$$

Hence

$$
\frac{\partial x}{\partial \hat{x}}=g_{0}, \quad \frac{\partial x}{\partial t_{i}}=g_{i}, \quad i \geq 1
$$

Therefore, by taking (2.14) into account, we have

$$
\frac{\partial G^{(n)}}{\partial t_{i}}=\left(\frac{\partial}{\partial t_{i}}+g_{i} \frac{\partial}{\partial x}\right) \frac{G^{(s)}}{g_{0}}=\frac{1}{g_{0}}\left\langle A_{i}^{(n)}+g_{i}, G^{(n)}\right\rangle=\left\langle A_{i}^{(s)}, G^{(s)}\right\rangle_{\hat{x}},
$$

where

$$
A_{i}^{(n)}=\lambda^{i}+\cdots+\hat{g}_{i-1} \lambda+\hat{g}_{i}, \quad \hat{g}_{i}:=\frac{g_{i}}{g_{0}},
$$

and $\langle U, V\rangle_{\hat{x}}:=U \partial_{\hat{x}} V-V \partial_{\hat{x}} U$.

Remark. This theorem shows that a correspondence $G^{(s)} \rightarrow G^{(n)}$ between the Schwarzian and normalized hierarchies exists. Reciprocally, a transformation $G^{(n)} \rightarrow G^{(s)}$ can also be defined provided that the solution $G^{(n)}=G^{(n)}(\lambda, x, \boldsymbol{t})$ does not vanish at $\lambda=0$. To prove this statement let us define

$$
u:=\frac{1}{\left.G^{(n)}\right|_{\lambda=0}},
$$

and the differential form

$$
\omega:=u\left(\mathrm{~d} x+\sum_{i \geq 1} g_{i} \mathrm{~d} t_{i}\right),
$$

where $g_{n}$ are the coefficients of the expansion of $G^{(n)}$ in powers of $\lambda$. From (1.1) it follows at once that

$$
\partial_{i} u=\left(u g_{i}\right)_{x}
$$

Moreover, from the identities

$$
\partial_{j} A_{i}-\partial_{i} A_{j}=\left\langle A_{j}, A_{i}\right\rangle,
$$


and taking into account that $g_{i}=\left.A_{i}\right|_{\lambda=0}$, we have

$$
\partial_{j} g_{i}-\partial_{i} g_{j}=\left\langle g_{j}, g_{i}\right\rangle
$$

and therefore

$$
\partial_{j}\left(u g_{i}\right)=\partial_{i}\left(u g_{j}\right)
$$

which proves that $\omega$ is a closed form. It is now straightforward to demonstrate that the function

$$
G^{(s)}(\lambda, \hat{x}, \boldsymbol{t}):=u(x, \boldsymbol{t}) G^{(n)}(\lambda, x, \boldsymbol{t}),
$$

where

$$
\hat{x}:=q(x, \boldsymbol{t}), \quad \omega=\mathrm{d} q,
$$

verifies the Schwarzian hierarchy (1.1).

\section{Reductions and differential constraints}

In [5] wide classes of reductions of the normalized hierarchy (1.1) were introduced by using the notion of compatible differential constraints. They can be easily generalized to the Schwarzian hierarchy as well. We will consider here reductions defined by second-order differential constraints

$$
2 G_{x x} G-G_{x}^{2}+4 a(\lambda)-4 U(\lambda, x, \boldsymbol{t}) G^{2}=0,
$$

where $a(\lambda)$ is an arbitrary function and

$$
U(\lambda, x, \boldsymbol{t}):=\left(\frac{a(\lambda)}{G^{2}}\right)_{+} .
$$

Requiring compatibility between (1.1) and (3.1) yields

$$
\partial_{n} U=-\frac{1}{2} A_{n, x x x}+2 U A_{n, x}+U_{x} A_{n}, \quad n \geq 1 .
$$

We notice that in terms of the generating function $H$ for the conservation laws, equations (3.1) and (3.2) can be written as

$$
\{H, x\}+a(\lambda) H^{2}=U(\lambda, x, \boldsymbol{t}), \quad U=\left(a(\lambda) H^{2}\right)_{+},
$$

where we are using the Schwarzian derivation operation

$$
\{H, x\}:=\frac{3}{4} \frac{H_{x}^{2}}{H^{2}}-\frac{1}{2} \frac{H_{x x}}{H} .
$$

The equations (3.1) determine integrable hierarchies associated with Schrödinger spectral problems with energy-dependent potentials. Thus, for $a(\lambda)=\lambda^{n}$ one finds the generalized $\mathrm{KdV}$ hierarchies for energy-dependent potentials of the form

$$
U(\lambda, x, \boldsymbol{t}):=\lambda^{n}+\sum_{i=0}^{n-1} \lambda^{i} u_{i}(x, \boldsymbol{t}), \quad \text { (normalized case) }
$$


and

$$
U(\lambda, x, \boldsymbol{t}):=\sum_{i=1}^{n} \lambda^{i} u_{i}(x, \boldsymbol{t}), \quad \text { (Schwarzian case) }
$$

For the normalized case $G=G^{(n)}$ the simplest choices $n=1$ and $n=2$ lead to the KdV and the Zakharov-Shabat hierarchies, respectively. We observe that for the Schwarzian case the choice $a(\lambda)=\lambda^{n}$ in (3.4) leads to the constraint (1.11).

Theorem 4. The differential constraint (3.4) is invariant under the transformation $H^{(s)} \rightarrow H^{(n)}$ determined by equation (2.16). That is to say, if $H^{(s)}$ satisfies

$$
\left\{H^{(s)}, x\right\}+a(\lambda) H^{(s) 2}=U^{(s)}(\lambda, x, \boldsymbol{t})
$$

then

$$
\left\{H^{(n)}, x\right\}+a(\lambda) H^{(n) 2}=U^{(n)}(\lambda, x, t) .
$$

The corresponding transformation law for the potential function is

$$
U^{(n)}=g_{0}^{2} U^{(s)}+\left\{g_{0}(\hat{x}), \hat{x}\right\}
$$

Proof. If we define $H^{(n)}:=H^{(s)} / h_{0}$, then it follows at once that

$$
\left\{H^{(s)}, x\right\}=h_{0}^{2}\left(\left\{H^{(n)}, \hat{x}\right\}+\frac{1}{4} \frac{h_{0, \hat{x}}^{2}}{h_{0}^{2}}-\frac{1}{2} \frac{h_{0, \hat{x} \hat{x}}}{h_{0}}\right) .
$$

On the other hand

$$
\left\{\frac{1}{h_{0}}, \hat{x}\right\}=\frac{1}{2} \frac{h_{0, \hat{x} \hat{x}}}{h_{0}}-\frac{1}{4} \frac{h_{0, \hat{x}}^{2}}{h_{0}^{2}} .
$$

Thus, it is straightforward to get

$$
\left\{H^{(n)}, \hat{x}\right\}+a(\lambda) H^{(n) 2}=U^{(n)}, \quad U^{(n)}:=\frac{1}{h_{0}^{2}} U^{(s)}+\left\{\frac{1}{h_{0}}, \hat{x}\right\} .
$$

A complete and convenient description of the general class of the energy-dependent hierarchies can be formulated by taking advantage of the identities (3.4). The following examples illustrate the fact that, in addition to the standard hierarchies of ZakharovShabat and KdV types, many other integrable models may be analyzed within the theory of the second order differential constraints of the Schwarzian hierarchy (1.1).

Example 1. For $a(\lambda)=\lambda$ and $G=G^{(s)}$, the constraint (3.4) reads

$$
\{H, x\}+\lambda H^{2}=\lambda u_{1},
$$

and by identifying the coefficient of $1 / \lambda$ we get

$$
\left\{h_{0}, x\right\}+2 h_{0} h_{1}=0 .
$$


Hence, by taking into account that in terms of the function $q:=q_{2}(x, \boldsymbol{t})$ of (2.15)

$$
h_{0}=\frac{1}{g_{0}}=q_{x}, \quad h_{1}=-\frac{g_{1}}{g_{0}^{2}}=q_{x} q_{t}, \quad\left(t:=t_{1}\right),
$$

we deduce at once the Schwarzian form of the KdV equation

$$
2 q_{t} q_{x}=\left\{q_{x}, x\right\} \Leftrightarrow-8 q_{t}+2 \frac{q_{x x x}}{q_{x}^{3}}-3 \frac{q_{x x}^{2}}{q_{x}^{4}}=0,
$$

which, written in terms of $g_{0}=1 / h_{0}$, reduces to the Harry-Dym equation

$$
g_{0, t}=\frac{1}{4} g_{0}^{3} g_{0, x x x} .
$$

Example 2. For $a(\lambda)=\lambda^{2}$ and $G=G^{(s)}$, the identity (3.4) reads

$$
\{H, x\}+\lambda^{2} H^{2}=\lambda^{2} u_{2}+\lambda u_{1},
$$

and by identifying the constant term in the expansion in powers of $\lambda$ we get

$$
\left\{h_{0}, x\right\}+h_{1}^{2}+2 h_{0} h_{2}=0 .
$$

Now, from (1.4) we have

$$
h_{0, t}=\left(\frac{h_{1}}{h_{0}}\right), \quad h_{1, t}=\left(\frac{h_{2}}{h_{0}}\right), \quad\left(t:=t_{1}\right) .
$$

Hence, by taking into account (3.9) we find

$$
\left(q_{x} q_{t}\right)_{t}=\left(\frac{1}{4} \frac{q_{x x x}}{q_{x}^{3}}-\frac{3}{8} \frac{q_{x x}^{2}}{q_{x}^{4}}-\frac{1}{2} q_{t}^{2}\right)_{x} .
$$

This nonlinear equation admits a Lagrangian given by

$$
\mathfrak{L}=\iint\left(q_{t}^{2} q_{x}+\frac{1}{4} \frac{q_{x x}^{2}}{q_{x}^{3}}\right) \mathrm{d} x \mathrm{~d} t
$$

or, equivalently, under the point transformation $q(u(y, t), t)=y$

$$
\mathfrak{L}=\iint \frac{u_{t}^{2}+u_{y y}^{2}}{u_{y}^{2}} \mathrm{~d} y \mathrm{~d} t .
$$

If we denote now $y \rightarrow x, u \rightarrow q$, the following equivalent Lagrangian formulation arises

$$
\begin{aligned}
\mathfrak{L} & =\iint\left(\frac{q_{t}^{2}+q_{x x}^{2}}{q_{x}^{2}}\right) \mathrm{d} x \mathrm{~d} t=\iint\left(\frac{\left(q_{t}+q_{x x}\right)^{2}}{q_{x}^{2}}-2 \frac{q_{t} q_{x x}}{q_{x}^{2}}\right) \mathrm{d} x \mathrm{~d} t \\
& =\iint \frac{\left(q_{t}+q_{x x}\right)^{2}}{q_{x}^{2}} \mathrm{~d} x \mathrm{~d} t .
\end{aligned}
$$

Thus, the model admits a Hamiltonian density

$$
H=q_{t} L_{q_{t}}-L=p_{x} q_{x}+\frac{1}{4} p^{2} q_{x}^{2}, \quad p:=L_{q_{t}}, \quad L:=\frac{\left(q_{t}+q_{x x}\right)^{2}}{q_{x}^{2}}
$$


which leads to the Hamiltonian equations

$$
q_{t}+q_{x x}+2 p q_{x}^{2}=0, \quad-p_{t}+p_{x x}=\left(2 p^{2} q_{x}\right)_{x} .
$$

It should be noticed that the last system is one of "canonical" forms of the isotropic Landau-Lifshitz model (see 9]).

In [5] first-order differential constraints defining reductions of (1.1) were also introduced. They adopt the form

$$
G_{x}-a(\lambda)-U(\lambda, x, \boldsymbol{t}) G=0,
$$

where $a(\lambda)$ is an arbitrary function and

$$
U(\lambda, x, t):=-\left(\frac{a(\lambda)}{G}\right)_{+} .
$$

Requiring compatibility between (1.1) and (3.15) gives

$$
\partial_{n} U=-A_{n, x x}+\left(U A_{n}\right)_{x}, \quad n \geq 1,
$$

or, equivalently,

$$
\partial_{n} U=-\partial_{x}\left(U\left(\lambda^{n} G\right)_{-}\right)_{+}, \quad n \geq 1, .
$$

In terms of the function $H$ equations (3.15) and (3.16) read

$$
H_{x}+a(\lambda) H^{2}+U(\lambda, x, \boldsymbol{t}) H=0, \quad U=-(a(\lambda) H)_{+} .
$$

We notice that for the Schwarzian case if we set $a(\lambda)=-\lambda^{m}$ in (3.15) the constraint (1.10) follows.

First-order differential constraints determine in turn further reductions of the secondorder differential constraints. Indeed, it follows easily that

Theorem 5. Given a solution $G$ of (3.15) then it satisfies

$$
2 G_{x x} G-G_{x}^{2}+a(\lambda)^{2}-4 \widehat{U}(\lambda, x, \boldsymbol{t}) G^{2}=0,
$$

where $U \rightarrow \widehat{U}$ is implemented by the Miura transformation

$$
\widehat{U}:=\frac{1}{2} U_{x}+\frac{1}{4} U^{2}
$$

Among the reductions defined by first-order differential constraints one finds the Burgers hierarchy as well as a class of hierarchies of energy-dependent type.

Example 3. If we impose on $G=G^{(n)}$ the constraint

$$
G_{x}=(\lambda+u) G-\lambda, \quad u=h_{1}=-g_{1},
$$

we get the Burgers hierarchy

$$
\partial_{n} u=\partial_{x}\left(\left(\partial_{x}+u\right)^{n} u\right) .
$$

In particular the flow corresponding to $t:=t_{1}$ is the Burgers equation

$$
u_{t}=u_{x x}+2 u u_{x}
$$


Example 4. For a constraint on $G=G^{(n)}$ of the form

$$
G_{x}=\left(\lambda^{2}+\lambda u_{1}+u_{0}\right) G-\lambda^{2}, \quad u_{1}=h_{1}=-g_{1}, \quad u_{0}=h_{2}=g_{1}^{2}-g_{2},
$$

we get the following hierarchy for the energy-dependent potential $U:=\lambda^{2}+\lambda u_{1}+u_{0}$

$$
\partial_{n}\left(\begin{array}{l}
u_{1} \\
u_{0}
\end{array}\right)=\left(\begin{array}{cc}
-\partial_{x} & 0 \\
0 & -\partial_{x}\left(\partial_{x}-u_{0}\right)
\end{array}\right) R^{n+1}\left(\begin{array}{l}
1 \\
0
\end{array}\right)
$$

where

$$
R:=\left(\begin{array}{cc}
-u_{1} & \partial_{x}+u_{0} \\
1 & 0
\end{array}\right)
$$

The flow corresponding to $t:=t_{1}$ reads

$$
u_{0, t}=u_{1, x x}-\left(u_{0} u_{1}\right)_{x}, \quad u_{1, t}=-\left(u_{1}^{2}+u_{0}\right)_{x},
$$

which in terms of the function $q:=q_{1}(x, t)$ of (2.15) reduces to

$$
q_{t t}-q_{x x x}-3 q_{x} q_{x t}-q_{t} q_{x x}+\left(q_{x}\right)_{x}^{3}=0 .
$$

It is possible to characterize multidimensional integrable models by imposing compatible differential constraints to (1.1). To illustrate this feature let us consider the constraint (3.20) for $G=G^{(n)}$ and denote $y:=t_{1}, t:=t_{2}$. Thus we have the system of equations

$$
\begin{aligned}
& G_{x}=\left(\lambda^{2}-\lambda g_{1}+g_{1}^{2}-g_{2}\right) G-\lambda^{2}, \\
& G_{y}=\left\langle\lambda+g_{1}, G\right\rangle, \\
& G_{t}=\left\langle\lambda^{2}+\lambda g_{1}+g_{2}, G\right\rangle .
\end{aligned}
$$

Let us introduce the function

$$
F:=u G=f_{0}+\frac{f_{1}}{\lambda}+\frac{f_{2}}{\lambda^{2}}+\cdots, \quad u:=\frac{1}{\left.G\right|_{\lambda=0}} .
$$

From (3.23) we get

$$
\begin{aligned}
& u_{x}=\left(g_{2}-g_{1}^{2}\right) u, \\
& u_{y}=\left(g_{1, x}+g_{1} g_{2}-g_{1}^{3}\right) u, \\
& u_{t}=\left(g_{2, x}+g_{2}^{2}-g_{2} g_{1}^{2}\right) u,
\end{aligned}
$$

so that the equations (3.23) written in terms of $F$ read

$$
\begin{aligned}
& F_{x}=\left(\lambda^{2}-\lambda g_{1}\right) F-\lambda^{2} f_{0}, \\
& F_{y}=\left(\lambda^{3}-\lambda g_{2}\right) F-\lambda^{3} f_{0}-\lambda f_{1}, \\
& F_{t}=\left(\lambda^{4}-\lambda g_{3}\right) F-\lambda^{4} f_{0}-\lambda^{3} f_{1}-\lambda^{2} f_{2} .
\end{aligned}
$$

Now it is easy to deduce from (3.24) that

$$
F_{t}=F_{x x}+2 g_{1} F_{y}-g_{1}^{2} F_{x}
$$


and, as a consequence, one finds that $v:=g_{1}$ and $w=\ln u$ satisfy the nonlinear system of equations

$$
\begin{aligned}
& v_{t}=v_{x x}+2 v v_{y}-v^{2} v_{x}+2 v_{x} w_{x}, \\
& w_{t}=w_{x x}+2 v w_{y}-v^{2} w_{x}+w_{x}^{2} .
\end{aligned}
$$

We observe that equations (3.24) mean that equation 3.25) and, consequently, equation (3.26) admit separation of variables. If we expand $F$ in powers of $\lambda$ then equations (3.24) become

$$
\begin{array}{ll}
f_{n, x}=f_{n+2}-g_{1} f_{n+1}, & f_{-1}=0, \quad f_{0}=u, \\
f_{n, y}=f_{n+3}-g_{2} f_{n+1}, & f_{2}=u g_{2}, \\
f_{n, t}=f_{n+4}-g_{3} f_{n+1}, & f_{3}=u g_{3} .
\end{array}
$$

By using (3.27a all the coefficients $f_{n}$ can be expressed as differential polynomials in $u$ and $v=g_{1}$ :

$$
f_{0}=u, \quad f_{1}=u v, \quad f_{2}=u_{x}+u v, \quad f_{3}=(u v)_{x}+v u_{x}+u v^{3}, \quad \ldots
$$

Furthermore, the system (3.27) implies that polynomial reductions of the form

$$
f_{n}=0, \quad \forall n \geq N,
$$

are Liouville integrable. Indeed, by noticing that $f_{N-1}=$ const, we require $N-1$ commuting vector fields to integrate such a reduction. For example, for $N=4$ the dynamical variables are $f_{0}, f_{1}, f_{2}$ and (3.27) can be written as

$$
\begin{aligned}
& \mathrm{d} f_{0}=\left(f_{2}-\frac{f_{1}^{2}}{f_{0}}\right) \mathrm{d} x+\left(\alpha-\frac{f_{1} f_{2}}{f_{0}}\right) \mathrm{d} y-\alpha \frac{f_{1}}{f_{0}} \mathrm{~d} t, \\
& \mathrm{~d} f_{1}=\left(\alpha-\frac{f_{1} f_{2}}{f_{0}}\right) \mathrm{d} x-\frac{f_{2}^{2}}{f_{0}} \mathrm{~d} y-\alpha \frac{f_{2}}{f_{0}} \mathrm{~d} t, \\
& \mathrm{~d} f_{2}=-\alpha \frac{f_{1}}{f_{0}} \mathrm{~d} x-\alpha \frac{f_{2}}{f_{0}} \mathrm{~d} y-\frac{\alpha^{2}}{f_{0}} \mathrm{~d} t,
\end{aligned}
$$

where $\alpha_{3}:=f_{3}=$ const. It is now straightforward to eliminate $\mathrm{d} x, \mathrm{~d} y, \mathrm{~d} t$ and find

$$
\begin{aligned}
& \mathrm{d} x=\mathrm{d}\left(\frac{f_{1}}{\alpha}-\frac{f_{2}}{2 \alpha^{2}}\right), \\
& \mathrm{d} y=\mathrm{d}\left(\frac{f_{0}}{\alpha}-\frac{f_{1} f_{2}}{\alpha^{2}}+\frac{f_{2}^{3}}{3 \alpha^{3}}\right), \\
& \mathrm{d} t=\mathrm{d}\left(-\frac{f_{0} f_{2}}{\alpha^{2}}-\frac{f_{1}^{2}}{2 \alpha^{2}}+\frac{f_{1} f_{2}^{2}}{\alpha^{3}}-\frac{f_{2}^{4}}{4 \alpha^{4}}\right),
\end{aligned}
$$

In this way the solution is determined by the following system

$$
\begin{aligned}
& f_{0}=\alpha y+x f_{2}-\frac{f_{2}^{3}}{2 \alpha}, \quad f_{1}=\alpha x+\frac{f_{2}^{2}}{2 \alpha}, \\
& -\frac{7}{8} f_{2}^{4}+\frac{1}{2} \alpha^{2} x f_{2}^{2}+\alpha^{3} f_{2}+\alpha^{4}\left(\frac{1}{2} x^{2}+t\right)=0 .
\end{aligned}
$$

This example shows the complicated algebraic singularities exhibited by the solutions of (3.26). 


\section{Acknowledgements}

A B Shabat wish to thank A Ibort for his support during his stay as a visiting professor of the Carlos III University of Madrid. He also acknowledges the group of the Department of Theoretical Physics II of the Complutense University for their warm hospitality. L Martinez Alonso was partially supported by the DGCYT project BFM2002-01607. A Shabat was partially supported by the RFFI project 01-01-00874 and Sc. Schools RF grant 0615-96093. Both authors are grateful to E Ferapontov for several useful and stimulating discussions.

\section{References}

[1] Rozdestvenskii B L and Sidorenko A D, On the Impossibility of "Gradient Catastrophe" for Weakly Nonlinear Systems, Comput. Math. \& Math. Phys. 7 (1967), 1176-1179.

[2] Dubrovin B A, Matveev V B and Novikov S P, Nonlinear Equations of Korteweg-de Vries Type, Finite-Band Linear Operators and Abelian Varieties, Usp. Mat. Nauk 31, Nr. 1 (1976), $55-136$.

[3] Mikhalev V G, Hamiltonian Formalism of Korteweg-de Vries-Type Hierarchies, Funct. Anal. Appl. 26, Nr. 2 (1992), 140-142.

[4] Hone A N W, Reciprocal Transformations, Painlevé Property and Solutions of EnergyDependent Schrödinger Hierarchies, Phys. Lett. A249 (1998), 46-54.

[5] Martinez Alonso L and Shabat A B, Energy-Dependent Potentials Revisited: A Universal Hierarchy of Hydrodynamic Type,Phys. Lett. A300 (2002), 58-64.

[6] Liouville J, J. Math. Pures Appl. 20 (1855), 137-138.

[7] Tsarev S P, Tomographical Methods in Physical-Technical Measurements, WNIIFTRI, Moscow, (1985) , 120-135.

[8] Ferapontov E V, Integration of Weakly Nonlinear Hydrodynamic Systems in Riemann Invariants, Phys. Lett. A158 (1991), 112-118.

[9] Adler V E, Marikhin V G and Shabat A B, Lagrangian Lattices and Canonical Bäcklund Transformations, Theor. Math. Phys. 129, Nr. 2 (2001), 163-183. 